\title{
Enhance in-Hand Dexterous Micro-Manipulation by Exploiting Adhesion Forces
}

\author{
Jean-Antoine Seon, Redwan Dahmouche, and Michaël Gauthier
}

\begin{abstract}
Micro-manipulation plays a key role in the development of complex and assembled micro-systems. However, current micro-manipulation solutions are often limited to small rotation amplitudes and to simple shaped objects (such as cubes). Our approach consists in developing in-hand micro-manipulation techniques using dexterous micro-hands to manipulate arbitrary shaped objects and to perform large rotations. This paper focuses on the trajectory generation of a dexterous micro-hand to achieve automated repositioning by taking advantage of adhesion forces. The results on the generated trajectories show that adhesion forces can be exploited to enhance the manipulation possibilities. Moreover, experiments show that planed rotations are performed at more than $95 \%$ using an open loop control. Dexterous micro-manipulation is a promising way to perform complex manipulation tasks in micro-scale.
\end{abstract}

Index Terms-Dexterous Manipulation, Micro/Nano Robots, Grasping, Manipulation Planning, Path Planning for Manipulators

\section{INTRODUCTION}

Dexterous manipulation has been an active field of research in macro-scale for decades [1]. Indeed, the grasping and manipulation planning problems have been explicitly formalized [2] and various approaches to perform dexterous manipulation have been studied such as rolling [3], sliding [4] and finger gaiting [5]. However, it has not been largely investigated in micro-manipulations which is usually limited to simple pick and place operations for simple objects [6] [7] [8] [9] [10]. We show in this paper that in-hand dexterous micro-manipulation is a promising way to control the rotation of micro-objects for micro-systems assembly.

In robotics, rotations can be obtained in two ways. The first one, which is the most common industrial solution, consists in using a basic tweezer placed on a robot which rotates the carried gripper. Thus, this approach uses simple gripper, such as a parallel jaw gripper, and the accuracy is limited by the backlash and the eccentricity in the robot's joints. The second way consists in using a dexterous hand to perform in-hand rotation [11] [12] [13] [14] [15]. This method, which requires more elaborated gripper, can be very versatile as a single "hand" can manipulate a large range of objects. The microhand fingers can be mounted on simple translation actuators with high repeatability. In this case, the accuracy is dependent on the contact between the fingers and the object surface.

Both approaches have different performances in microscale. Indeed, backlash and eccentricity (typically around $10 \mu \mathrm{m}$ [16]) might be comparable to the object dimensions

The authors are with FEMTO-ST Institute, AS2M department, Univ Bourgogne Franche-Comté, CNRS, 24 rue Savary, 25000 Besançon, France. ja.seondfemto-st.fr, redwan.dahmouchedfemto-st.fr, michael.gauthierdfemto-st.fr (typically around $1 \mu \mathrm{m}$ to $100 \mu \mathrm{m})$. Consequently, in the first way, the accuracy is highly disturbed by backlash and eccentricity in the robot joints. Thus, this paper focuses on the second way proposing to perform micro-object rotation using in-hand dexterous manipulation.

Dexterous manipulation in micro-scale is different from the macro-manipulation since gravitational and inertial forces are dominated by the surface forces such as van der Waals, electrostatic and capillary forces [17]. These attractive forces change the manipulation paradigm as the objects stick to both the manipulating fingers and the substrate. Thus planning finger trajectory for effective dexterous manipulation in microscale is significantly different from macro-scale.

Two main approaches might be considered to take into account these particularities. The first one consists in modifying the contact properties to reduce adhesion forces by varying environmental parameters such as humidity and temperature [18] or by controlling the surface roughness [19]. These methods are used to get as close as possibleto macro-scale manipulation. The second one consists in developing new strategies taking into account these adhesion forces. Indeed, it has been shown that taking advantage of these forces can enhance the gripping strategies [20]. Moreover, even if the adhesion phenomena is not fully predictable, creating sticky fingers is possible using nanostructuration [21] or chemical functionalization [22].

In this paper we propose to exploit these adhesion forces, to enhance the stability and the strategies of micro-manipulation. The main contribution of this paper is the development of the first trajectory planner for in-hand dexterous micromanipulation that takes into account adhesion forces. Original fingers' trajectories are proposed and the benefit of exploiting adhesion forces is demonstrated. The content of this paper has been partially mentioned in a previous conference paper [23]. This article presents a more detailed methodology and trajectories validated experimentally considering different conditions of use.

The next section gives an overview of the related work in automatic dexterous micro-manipulation while Section III gives a general formalization of this problem. Section IV details our methodology to compute stable grasps and generate trajectories. Finally, Section V presents several trajectories for in-hand manipulation with sticky fingers which are compared to classical non-sticky finger trajectories.

\section{RELATED WORK}

One of the first works on dexterous micro-manipulation was conducted by Thompson and Fearing on the orthotweezers [24]. In this system, two orthogonal fingers were 

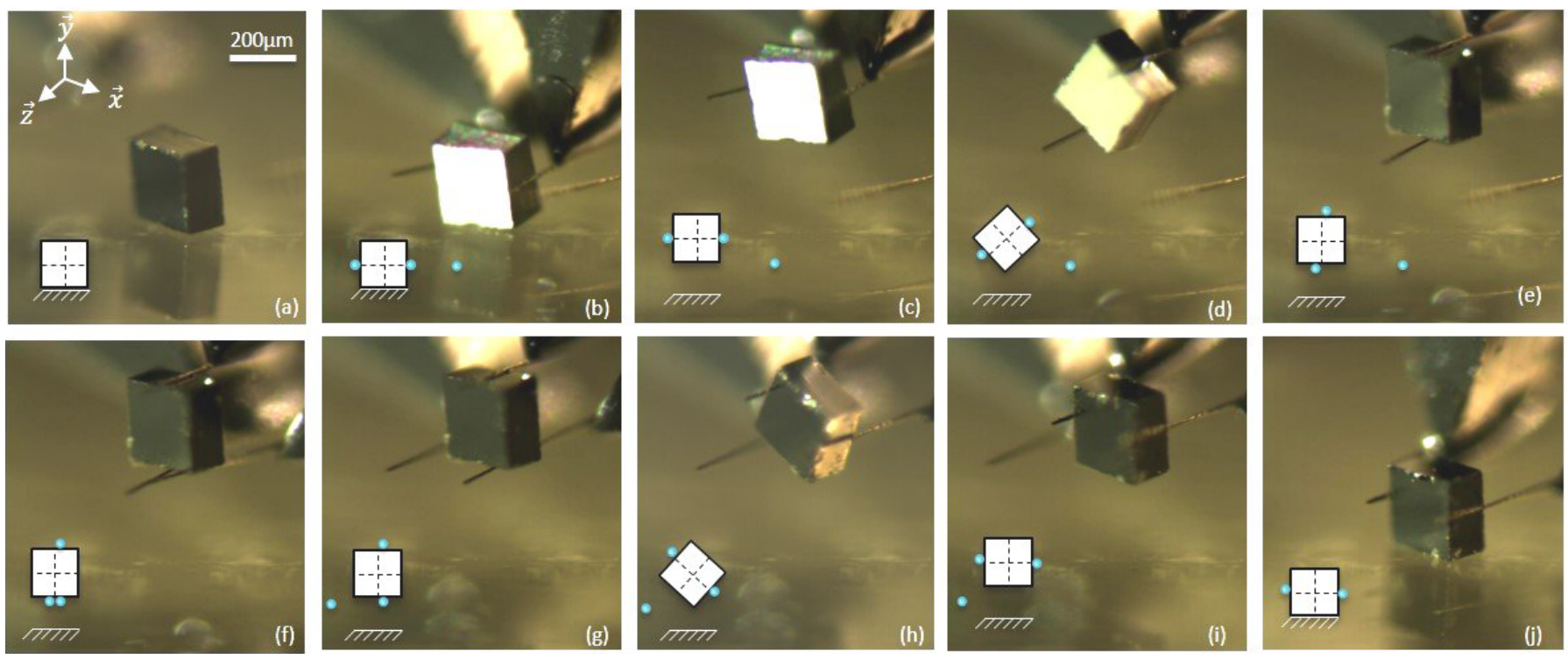

Fig. 1: Illustration of an automatic $180^{\circ}$ rotation performed using 3 fingers on our manipulation setup [13].

used to manipulate micro-blocks $(200 \mu \mathrm{m} \times 200 \mu \mathrm{m} \times 100 \mu \mathrm{m})$. The manipulation process was performed using manipulation primitives such as grasp the object, rotate along z-axis, etc. Since a two fingers hand has limited dexterity, a static external block was used to achieve a two axis rotation.

In 2006, Zhou et al. developed a 6 degrees of freedom (DOF) manipulator able to perform automatic in-hand manipulation [11]. The manipulator used only two fingers to manipulate micro-blocks $(300 \mu \mathrm{m} \times 300 \mu \mathrm{m} \times 100 \mu \mathrm{m})$. Similarly, Wason et al. have worked on automated micro-assembly using three probes [26]. Two of them were used to grasp the object while the last probe was passive and was used to generate out of plane rotations for polygonal objects. In these methods, fingers' rolling on the object during the rotation was neglected and no finger gaiting was used which limits the rotation amplitude.

In 2015, Zhan et al. developed a planar micro-gripper able to accurately control the orientation of optical fibers [25]. This micro-manipulator was composed of two jaws designed specifically to rotate optical fibers over $90^{\circ}$. This micromanipulation is performed thanks to the rolling of the optical fiber when the two jaws translate. However, this setup is limited to cylindrical shaped objects and the rotation is limited because of the number of jaws.

Recently, Brazey et al. have developed a dexterous micromanipulation system able to perform large rotations of microobjects [13]. The setup is composed of three fingers having two degrees of freedom each. Fig. 1 shows a $180^{\circ}$ rotation of a $200 \mu \mathrm{m}$ micro-square. The manipulation process is fully automated and is based on trajectories generated using manipulation primitives.

Beyond the current dexterous micro-manipulation approaches, the proposed method developed in this paper is not limited to squared objects. Indeed, arbitrary shapes are considered and the rolling constraint of the fingers during the object rotation is explicitly taken into account. Furthermore, the sticky forces that may exist in the micro-scale are taken into account in the finger path planning.

\section{Modeling AND BACKGROUND}

This section formalizes the grasping and in-hand manipulation problem in micro-scale and emphasizes the contact modeling differences with the macro-scale.

\section{A. Grasping Forces}

Let us consider the general case of six DOF manipulation using $N$ fingers. In order to manipulate the object, fingers must apply a grasping force on the object's surface. This action can be modeled in three ways: i) punctual and frictionless contact, ii) punctual and frictional contact, iii) soft contact. We assume that, in micro-scale, the applied forces can be modeled using the punctual frictional model. and thus using the Coulomb law. This means that, no slippage happens as far as the applied force lies in a three dimensions cone:

$$
\sqrt{f_{t_{1}}^{2}+f_{t_{2}}^{2}} \leq \mu f_{n}
$$

where $f_{t_{1}}$ and $f_{t_{2}}$ are tangential components of the force, $f_{n}$ is the normal component and $\mu$ is the friction coefficient.
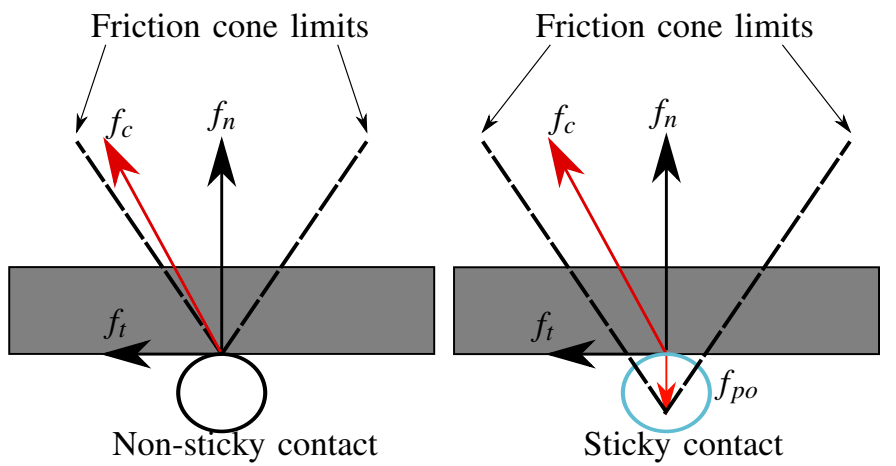

Fig. 2: Impact of pull-off force on the friction cone. 
In the case of micro-manipulation, this contact law is slightly modified. Indeed, adhesion acts as an attractive force $\left(f_{p o}\right)$ between the finger and the manipulated object. This force, called pull-off force, represents the force required to detach the finger from the object. In presence of this force, the Coulomb law can be rewritten as follows:

$$
\sqrt{f_{t_{1}}^{2}+f_{t_{2}}^{2}} \leq \mu\left(f_{n}+f_{p o}\right)
$$

The effect of this attractive force on the slippage limit condition is that the friction cone is shifted as depicted in Fig.2. In addition, contrary to the macro-scale, where only positive grasping forces can be applied (push the object), in micro-scale, it is possible to apply negative forces (pull the object) as long as the force lies in the modified friction cone.

\section{B. Equilibrium}

During all the manipulation process, the object must be in equilibrium. A $N$ fingers grasp is in static equilibrium if and only if the following equation is satisfied:

$$
-w_{\text {ext }}=G \cdot f_{c}=\sum_{i=1}^{N} w_{c_{i}}
$$

where $w_{\text {ext }}$ is the external wrench (force and torque) applied to the object, the matrix $G \in \mathfrak{R}^{6 \times 3 N}$ is called the grasp matrix [27] which depends on the contact positions, $f_{c} \in \mathfrak{R}^{3 N}$ is the vector containing the $N$ grasping forces and $w_{c_{i}} \in \mathfrak{R}^{6}$ is the $i^{\text {th }}$ grasping wrench.

Given a set of contact points, the equilibrium problem consists in finding a set of grasping forces $f_{c}$ which verify equation (3) and the non-slippage constraint defined by (2). This problem can be rewritten using the limits of the friction cones. Indeed, the grasping wrench $w_{c_{i}}$ applied at the $i^{t h}$ contact point is a linear combination of wrenches that approximate the cone:

$$
\left\{\begin{array}{c}
w_{c_{i}}=\sum_{j} \alpha_{i, j} \cdot w_{l_{j}, i}+\beta_{i} \cdot w_{p o_{i}} \\
\alpha_{i, j} \geq 0 \\
1 \geq \beta_{i} \geq 0
\end{array}\right.
$$

where $w_{l_{j}, i}$ is one wrench that approximate the $i^{\text {th }}$ friction cone, $w_{p o_{i}}$ is the wrench induced by pull-off forces (sticking effect), and $\alpha_{i, j}$ and $\beta_{i}$ are coefficients that must be positive to stay inside the friction cone. Note that in macro-manipulation, $w_{p o_{i}}=0$. Then, the equilibrium problem of an $N$ fingers grasp (3) can be rewritten as a function of the $N$ friction cones wrenches:

$$
\left\{\begin{array}{c}
-w_{e x t}=\sum_{i=1}^{N} w_{c_{i}}=\sum_{i=1}^{N}\left(\sum_{j} \alpha_{i, j} \cdot w_{l_{j}, i}+\beta_{i} \cdot w_{p o_{i}}\right) \\
\alpha_{i, j} \geq 0 \\
1 \geq \beta_{i} \geq 0
\end{array}\right.
$$

Thus, the equilibrium problem is equivalent to finding a set of positive coefficients $\left(\alpha_{i, j}, \beta_{i}\right)$ (not all of them null) verifying the previous equation. The equilibrium property can be characterized using the convex hull formed by the friction cones wrenches $\left(w_{l_{j}, i}\right)$. Indeed, it has been proven in [28] that, without external perturbation and any adhesion forces, if this convex hull contains the origin of the wrench space, then a solution to the equilibrium problem exists (as depicted in Fig.3). In fact, the grasp can exert all the wrenches that lie inside the convex hull. When the pull-off forces exist, the convex hull is formed by the friction cones wrenches $\left(w_{l_{j}, i}\right)$ and the adhesion wrenches $\left(w_{p o_{i}}\right)$.

Since inertial forces of micro-objects are negligible, the manipulation process is a succession of static equilibrium states that are verified along the manipulation trajectory.

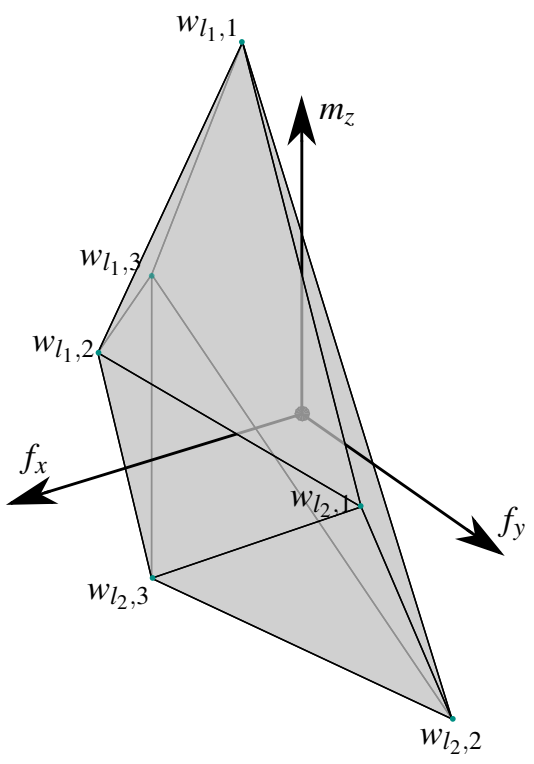

Fig. 3: Representation of a convex hull formed by three nonsticky fingers for a planar problem (six wrenches with three coordinates). The origin is included in this envelop thus the grasp is stable.

\section{Pull-off Forces and Finger Gaiting}

During finger gaiting (i.e. detaching a finger from the object and eventually repositioning it), the pull-off force between the fingers and the object has an important impact. Indeed, when re-grasping, the finger pull the object with a measured value $w_{p o}$. This pull-off force may disturb the grasping equilibrium. In other words, the remaining grasping fingers must compensate for the pull-off force caused by the removed finger to guarantee the object's stability. This can be formalized as follows:

$$
-w_{\text {ext }}-w_{p o}=G \cdot f_{c}=\sum_{i=1}^{N-1} w_{c_{i}},
$$

where $w_{p o}$ is the wrench induced by the release of the $\mathrm{N}^{t h}$ contact.

Considering (6), the re-grasping problem consists in finding positive $\left(\alpha_{i, j}, \beta_{i}\right)$ verifying the following equation:

$$
\left\{\begin{array}{c}
-w_{e x t}-w_{p o}=\sum_{i=1}^{N-1} w_{c_{i}}=\sum_{i=1}^{N-1}\left(\sum_{j} \alpha_{i, j} \cdot w_{l_{j}, i}+\beta_{i} \cdot w_{p o_{i}}\right) \\
\alpha_{i, j} \geq 0 \\
1 \geq \beta_{i} \geq 0
\end{array}\right.
$$


Similarly to the equilibrium problem, it is possible to use the convex hull formed by the $N-1$ remaining fingers to test if the grasp is stable during finger gaiting. In this case, if the external perturbation $\left(-w_{e x t}-w_{p o}\right)$ is included in the hull, then the grasp is stable.

The next section introduces a trajectory planer for dexterous manipulation which fulfills the constraints (5) and (7)

\section{Manipulation Planning}

We assume that the manipulation is performed using cylindrical fingers and that the geometry of the object is known, meaning that the object's surface is represented by a discrete regular sampling. We propose to generate the finger trajectories based on a two steps method. Given an object shape and the number of fingers of the manipulation system, the first step of the trajectory generation consists in computing the set of all stable grasps and admissible finger gaiting configurations. This step can be achieved off-line and is done only once for a given object. The second step consists in navigating between these configurations to define a path from the initial configuration to the desired one.

\section{A. Equilibrium and Re-Grasping Maps}

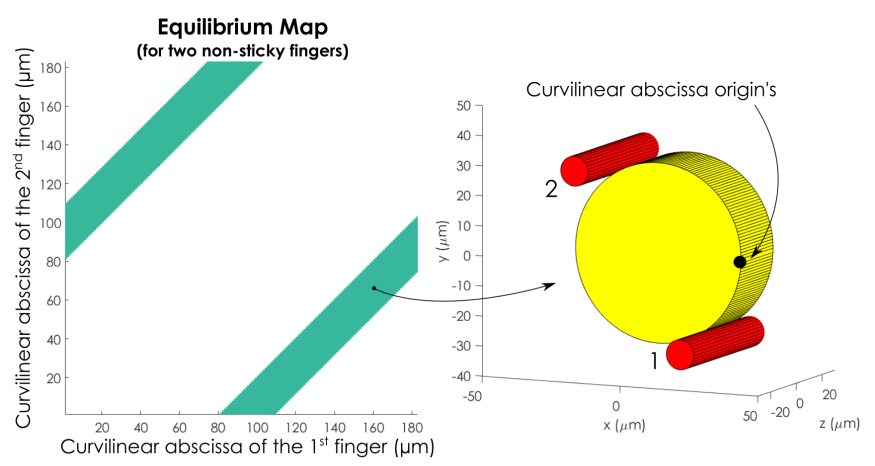

Fig. 4: Representation of the set $M_{2}$ for a 2D object, without considering adhesion (non-sticky behavior, $\forall i, w_{p o_{i}}=0$ ).

The maps representing the set of equilibrium grasps during the different steps of the manipulation process are obtained by testing, for every grasping configuration, if the convex hull formed by the friction cones wrenches $\left(w_{l_{i, j}}\right.$ and $\left.w_{p o_{i}}\right)$ contains the zero wrench. When external perturbations are considered, the existence of a solution, $\left(\alpha_{i, j}, \beta_{i}\right)$ respecting (5), consists in testing if the convex hull contains the external wrench. Thus, the equilibrium map, corresponding to the stable $\mathrm{N}$-fingers grasps, can be formalized as:

$$
\begin{aligned}
M_{N}= & \left\{c=\left(c_{1}, . ., c_{N}\right) \in \Re^{(3 \times N)} \mid\right. \\
& \left.-w_{\text {ext }} \in \operatorname{Convhull}\left(w_{l_{1}, 1}, \ldots, w_{l_{j}, N}, w_{p o_{1}}, \ldots, w_{p o_{N}}\right)\right\},
\end{aligned}
$$

where $c$ is a vector containing the contact coordinates on the object surface and Convhull $\left(w_{l_{1}, 1}, \ldots, w_{l_{j}, N}, w_{p o_{1}}, \ldots, w_{p o_{N}}\right)$ represents the convex hull.

Fig.4 depicts the $M_{2}$ map in the case of a cylindrical object without external forces $\left(w_{\text {ext }}=0\right)$. We chose to use the curvilinear abscissa as coordinates of the contact point in order to better illustrate the maps. The colored areas on the left figure represent the equilibrium configurations for a two fingers grasp, without any pull-off forces. Since permuting the two contact points leads to the same grasp, the equilibrium map shows a symmetry axis. Physically, the strip's width depends on the angular aperture of the friction cone.

In finger gaiting, to guarantee that a given finger $i$ can be pulled-off from the object without disturbing the grasp, we ensure that the resulting wrench $w_{p o_{i}}$, induced by the pulloff force $f_{p o_{i}}$, is included in the convex hull formed by the friction cones' wrenches of the $N-1$ remaining contacts. The re-grasping map, corresponding to the detachment of the $i^{\text {th }}$ finger in an $N$-fingers grasp, can be formalized as follows:

$$
\begin{aligned}
D_{N_{i}}= & \left\{c=\left(c_{1}, . ., c_{i}, . ., c_{N}\right) \in \mathfrak{R}^{(3 \times N} \mid\left(c_{1}, . ., c_{i-1}, c_{i+1},\right.\right. \\
& \left.. ., c_{N}\right) \in M_{N-1},-w_{e x t}-w_{p o, i} \in \operatorname{Convhull}\left(w_{l_{1}, 1}, . ., w_{l_{j}, i-1},\right. \\
& \left.\left.w_{l_{j}, i+1}, . ., w_{l_{j}, N}, w_{p o_{1}}, . ., w_{p o_{i-1}}, w_{p o_{i+1}}, . ., w_{p o_{N}}\right)\right\} .
\end{aligned}
$$

Similarly to the equilibrium maps, permuting the contact points leads to the same grasp thus the re-grasping map corresponding to the detachment of a finger are simply noted $D_{N}$ with $N \geq 2$.

Fig.5 (left) shows all the re-grasping configurations for an assigned location of the removed contact in the case of a three fingers grasp (fingers are sticky). For instance, in the configuration represented in Fig.5 (right), removing finger 3 would disturb the grasp's equilibrium.

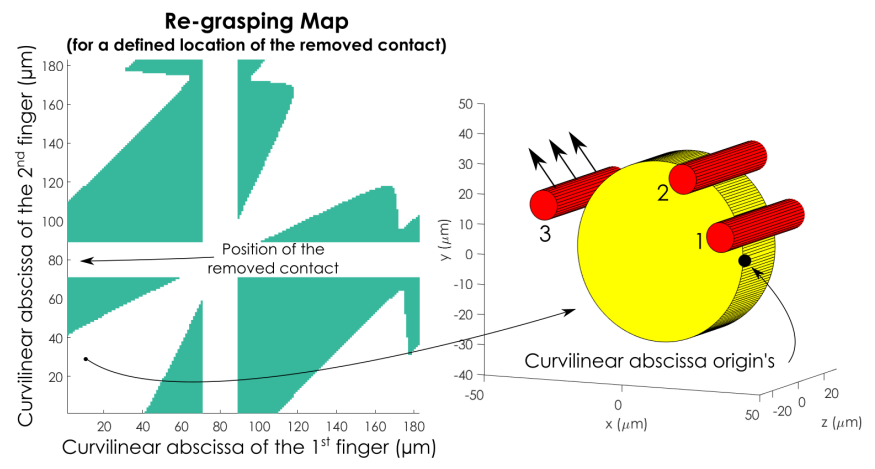

Fig. 5: Right: representation of the set $D_{3}$ for an assigned location of the removed contact (curvilinear abscissa of finger 3: $80 \mu \mathrm{m}$ ) considering adhesion (sticky behavior, $\forall i, w_{p o_{i}} \neq 0$ ). Left: example of a configuration outside $D_{3}$ in which finger 3 cannot be removed.

In addition, when the pull-off force between the object and the substrate is not negligible, we have to consider a third set of grasps that represents the initial grasps enabling to detach the object from the substrate (resist to the external wrench $w_{p o, s u b}$ induced by the substrate) and stably pick-up the object. This can be formalized as follows:

$$
\begin{aligned}
I_{N}= & \left\{c=\left(c_{1}, . ., c_{N}\right) \in \mathfrak{R}^{(3 \times N)} \mid\left(c_{1}, . ., c_{N}\right) \in M_{N},\right. \\
& \left.-w_{\text {ext }}-w_{\text {po }, \text { sub }} \in \text { Convhull }\left(w_{l_{1,1}}, \ldots, w_{l_{N, j}}, w_{p o_{1}}, \ldots, w_{p o_{N}}\right)\right\} .
\end{aligned}
$$


These $I_{N}$ maps are very similar to the previous maps but are just used to select the admissible initial grasp when the substrate is sticky.

\section{B. Planning Object Rotation}

1) Representation: Considering a discrete regular representation of the object, all the equilibrium maps $M_{k}(1<k<N)$ can be seen as a graph where every equilibrium position is a node. The goal is to navigate inside and between the maps to reach the desired object pose. In fact, navigating through a $M_{k}$ map describes the manipulation of the object with rolling contacts whereas navigating between two maps ( $M_{k}$ and $M_{k \pm 1}$ ) characterizes re-grasping. In this case, the regrasping maps $D_{k}$ are also part of the graph. Each node in $D_{k}$ is specifically used to link two adjacent sets $\left(M_{k}\right.$ and $\left.M_{k-1}\right)$ when a finger is removed. Conversely, as adhesion does not perturb the grasp when adding a finger, the link from $M_{k}$ to $M_{k+1}$ is systematically established.

2) Navigation Constraints: Three constraints must be taken into account when navigating inside and between the maps. The first one is the rolling constraint and thus the navigation in the $M_{k}$ maps. Indeed, in order to manipulate an object with $N$ fingers considering rolling without sliding, all the fingers must roll the same distance and in the same direction on the object surface. Consequently, the rolling constraint induces a unique available path in a $M_{k}$ map (depending on the radius of the fingers). Thus, each node in $M_{k}$ has a maximum of two neighbors in the same map. As an example, we consider the map and the grasp depicted in Fig4. The grasp corresponds to the element $M_{2}(160,65)$ in which the fingers have the same radius. Thus, the neighbors of this grasp are $M_{2}(160+\delta, 65+$ $\delta$ ) and $M_{2}(160-\delta, 65-\delta)$ (with $\delta$ representing the step of the discrete representation). Indeed, for a clockwise rotation the next grasp will be $M_{2}(160-\delta, 65-\delta)$ otherwise the next grasp will be $M_{2}(160+\delta, 65+\delta)$.

The second constraint is related to removing a finger. In this case, a node $A$ in $M_{k}$ is linked to a maximum of $k$ elements in $M_{k-1}$ corresponding to the possible removal of each of the $k$ fingers. For instance, when changing from a three fingers grasp to a two fingers grasp, a maximum of three neighbors are possible. However, this link is established only if the finger can be removed meaning that node A is in $D_{k}$. In other words, a node $A$ in $M_{k}$ is linked to a node $B$ in $M_{k-1}$ if and only if $A$ is included in $D_{k}$ and if the $k-1$ fingers used in $B$ are also used in $A$.

The last constraint deals with adding a finger. To switch from $M_{k}$ to $M_{k+1}$, it is required that the $k$ fingers used to form the grasp in $M_{k}$ are also used in the grasp with $k+1$ fingers (obviously at the same position on the object surface).

The collisions between the fingers are also tested in order to guarantee that the generated trajectory is reachable by the manipulator. In addition, the fingers cannot have two contact points or more with the object's surface. Thus, depending on the object geometry and on the finger radius, some nodes in $M_{k}$ and $D_{k}$ are discarded. Moreover, in the case of polygonal objects, we consider that the fingers cannot be positioned on the vertices.
3) Trajectory Generation: This operation consists in navigating in the generated maps using the $A^{*}$ algorithm. This heuristic graph search algorithm provides a complete and optimal path between the initial and the goal node. $A^{*}$ has been used in micro-manipulation [29] and also in micro-assembly [30] but, based on our knowledge, it has never been used for planning in-hand micro-manipulations. We chose to implement an $A^{*}$ algorithm for our trajectory planner in order to obtain optimal manipulation trajectories.

4) Algorithm Characteristics: An important parameter of the graph search is the cost function used to characterize the distance between two nodes. Considering the way we can navigate through the graph, we define three cost functions corresponding to the three possible actions (rolling, placing a finger on the object, removing a finger).

For the first case (rolling), the cost function is defined as the rolling distance needed to go from the current node to the next one. This distance is the arc length between two adjacent positions on the object surface and is noted $L_{\text {roll }}$.

As the fingers might be compliant, the fingers base displacement achieved by the actuator to detach the object from the object depends of the finger stiffness, its length and its radius. The cost, $C_{r}$, for detaching a finger from the object corresponds to the minimal distance applied by the actuator to guarantee that the finger is detached from the object.

For the last action (adding a finger), the cost, $C_{a}$, corresponds to an approximation of the distance between the last position of the finger and the new one. This value represents an approximation of the distance required to put in contact the finger with the object.

Moreover, the $A^{*}$ algorithm requires defining an admissible heuristic. The heuristic is defined as the remaining rotation from the current node to the desired one. Moreover, as rolling is used to rotate the object, the heuristic can be defined as a distance. Indeed, considering that the finger used for the manipulation has a radius $r_{d}$ and that rot is the rotation amplitude in radians, the heuristic will be $d_{r o t}=r_{d}$.rot at the initial node. Obviously this heuristic never overestimates the distance to the goal so it is an admissible heuristic for the considered costs.

\section{Planning Finger Gaiting}

Planning fingers trajectories during finger gaiting can be achieved using the same algorithm presented above $\left(A^{*}\right)$. Indeed, in this case, the nodes correspond to the spatial coordinates and the algorithm must find a path between the initial finger position and the new desired position by avoiding collisions with the object and the other fingers.

1) Displacement Process: Three displacement processes are considered: (i) remove a finger, (ii) place a finger and (iii) change the contact position of a finger.

As depicted in Fig.6, the release of a finger must be divided into two phases. The first one, which is the most critical, consists in precisely detach the finger from the object. Considering that $P_{c}$ is the coordinates of the finger when it is in contact with the object, the first step consists in moving from $P_{c}$ to a new position $P_{a, c}$ which corresponds 
to a position where the finger is clearly detached from the object. Thus, we arbitrarily chose to locate this new position at a distance of $2 \times r_{d}$ along the normal of the contact point ( $P_{a, c}=P_{c}-2 \times r_{d} \vec{N}_{c}$ where $\vec{N}_{c}$ is the normal to the object at the contact point). Obviously, this position depends on the finger's characteristics such as stiffness and length. The second phase consists in reaching the initial position $P_{0}$ by moving freely in the space.

Similarly, placing a finger is divided into two phases. The first step consists in moving freely in the space from $P_{0}$ to a position near the new contact $P_{a, c}$. Then, the second step, consists in moving along the contact's normal to precisely place the finger on the object.

In order to change the contact position, the finger must be detached from the current contact position $P_{c_{1}}$ by moving to $P_{a, c_{1}}$. Then the finger moves freely from $P_{a, c_{1}}$ to a position near the new contact $P_{a, c_{2}}$. Finally, the finger is placed on the object by moving from $P_{a, c_{2}}$ to the contact position $P_{c_{2}}$.
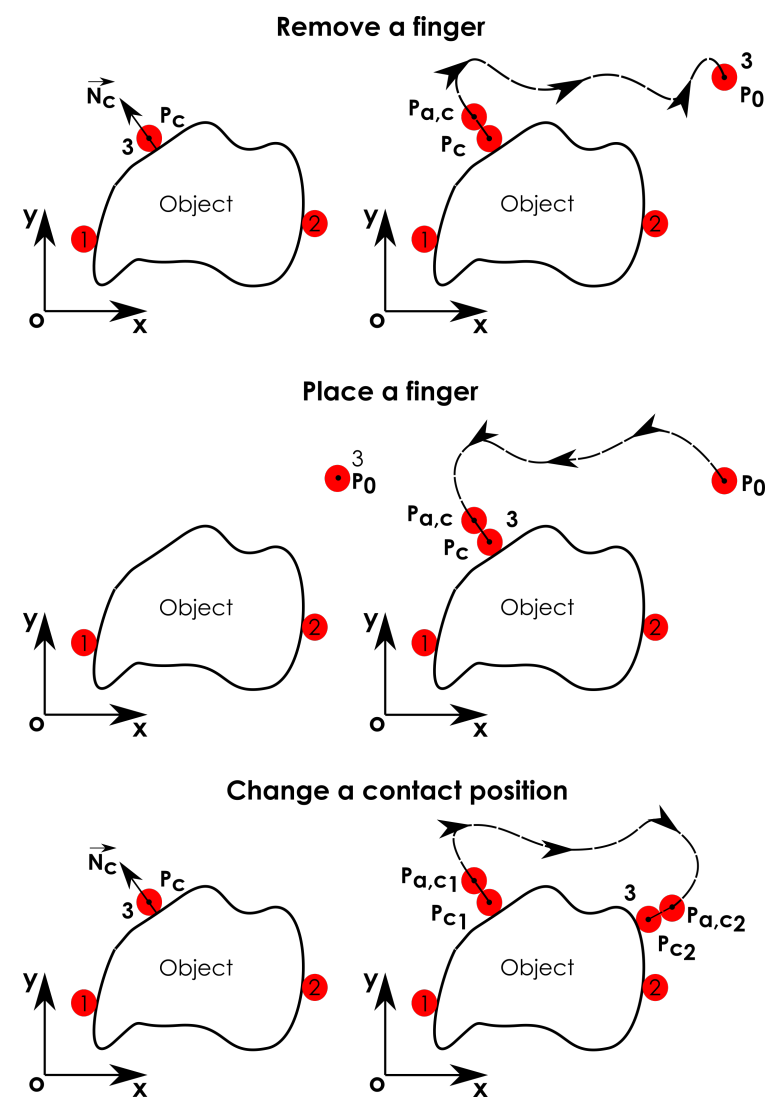

Fig. 6: Illustration of the three displacement process used for a finger gaiting step. Left images describe the initial state while right images represent the final state and an example of trajectory. The depicted trajectories are not the optimal trajectories.

2) Algorithm Characteristics: For these trajectories, there is only one cost function linked to the displacement of the finger in the space. Thus this displacement is evaluated by using the Euclidean distance between two points.

Moreover, the heuristic is chosen as the Euclidean distance between the current position and the goal position. Again, this heuristic never overestimates the distance to the goal so it is an admissible heuristic for the considered cost function.

\section{RESUlts}

The methodology presented in the previous section has been implemented and tested to generate trajectories for planar manipulation. In fact, in micro-assembly, most of the objects made using micro-fabrication techniques are planar. The results presented in this section are thus applicable in microassembly.

The results below present trajectories for two types of objects (arbitrary shaped and polygonal) and in two different conditions of manipulation (with and without sticky fingers). Note that every initial grasp is taken randomly in the set of admissible initial grasps. Furthermore, different finger's bases are tested to highlight the fact that our algorithm is suitable for several planar manipulation setups.

Moreover, the translation of the object is simply performed by the translation of all the fingers which does not affect the stability of the grasp. Thus, we will focus in this study on the rotations.

\section{A. Sticky Fingers}

These simulations focus on illustrating the trajectory generation when considering sticky fingers. We consider the manipulation with a three fingers hand where each finger can translate in the plan. This means that all the possible grasps and in-hand manipulation are represented by three maps: $M_{1}$, $M_{2}, M_{3}$. Two maps $\left(D_{2}\right.$ and $\left.D_{3}\right)$ are used for re-grasping and finger gaiting.

In these study cases, we consider the following cost functions: $C_{a}=150 \mu \mathrm{m}, C_{a_{r}}=8 \mu \mathrm{m}$ and $C_{r}=10 \mu \mathrm{m}$.

1) Arbitrary Shaped Object: Fig.7 illustrates the computed trajectory for a $132^{\circ}$ rotation using fingers with a radius of $5 \mu \mathrm{m}$. By choosing a suitable substrate material the pull-off force between the object and the substrate can be neglected. The pull-off force between fingers and the object and the friction coefficient are respectively estimated at $0.6 \mu \mathrm{N}$ and 0.3 . The weight of the object is neglected which means that the external wrench is null $\left(w_{\text {ext }}=0\right)$. In this setup, we consider that the fingers are attached to a rectangular moving base.

Fig.7a represents the initial grasping configuration used to pick-up the object while Fig.7b to Fig.7f show the rotation. As depicted in Fig.7e, the top finger is removed to avoid multiple contact between the object and the finger. This manipulation sequence, which is stable thanks to the adhesion, cannot be reproduced without adhesion (see Fig.7b for example).

2) Polygonal Object: The generated trajectories for a polygonal object are described in Fig.8. This $206^{\circ}$ rotation has been done considering finger with a radius of $5 \mu \mathrm{m}$, a negligible pull-off force between the object and the substrate and a pull-off force between the object and the fingers of $1.5 \mu \mathrm{N}$. The friction coefficient is again taken constant $(0.26)$ and the object's weight is again neglected. In this setup, we consider that the fingers are attached to triangular base as shown in Fig.1. 

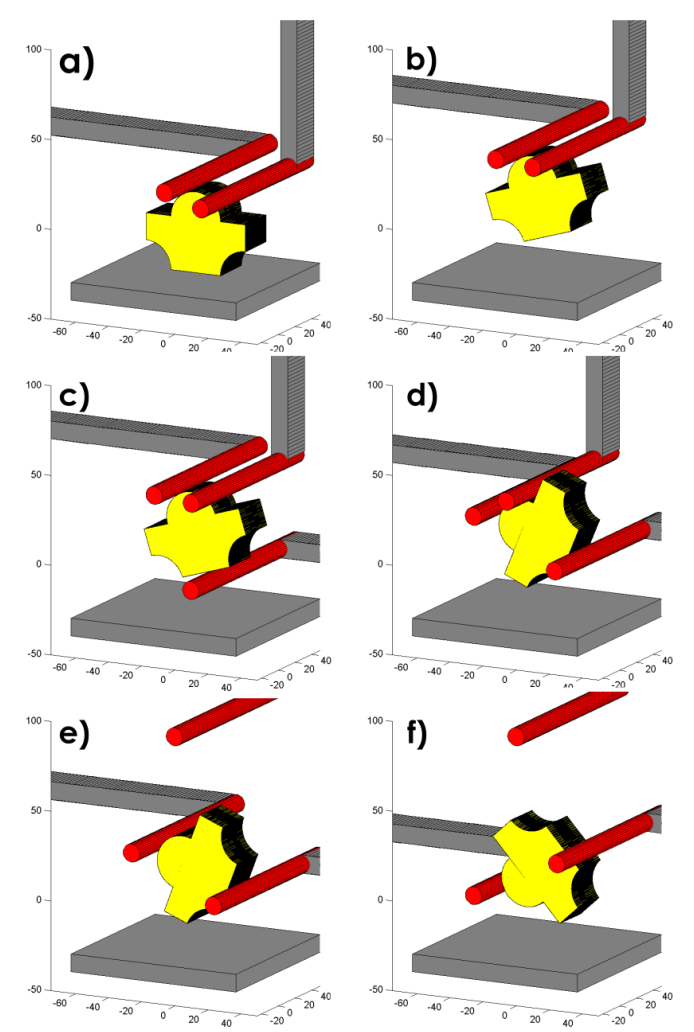

Fig. 7: Images sequence describing the trajectory generated by the planner for a $132^{\circ}$ rotation with sticky fingers: a) represents the initial grasping configuration while b) to f) represent the rotation steps. A finger gaiting step occurs in e) to avoid multiple contact between the object and the finger.

In this case, the manipulation process requires no finger gaiting steps. Thanks to adhesion properties, this high rotation amplitude can be performed using only two fingers. Obviously, these manipulation trajectories are not stable without using sticky fingers.

\section{B. Non-sticky Fingers}

In order to illustrate the impact of adhesion on the fingers' trajectories, we consider the same simulation but without any pull-off forces $\left(f_{p o}\right)$ between fingers and the object.

1) Arbitrary Shaped Object: Fig.9 illustrates the computed trajectory for a $132^{\circ}$ rotation. Contrarily to the sticky case which required only one finger gaiting step, this rotation required three finger gaiting steps. Indeed, Fig 9c shows the first one where the right finger is added because the two other fingers have reach the limits of the friction cones. Then in Fig 9e the left finger is repositioned to avoid the vertices. Finally, in Fig 9g, the top finger is also removed to avoid the vertices. Thus, without sticky fingers, the same manipulation requires more steps to be achieved.

2) Polygonal Object: As in the previous case, a $206^{\circ}$ rotation of the polygonal object is performed (see Fig.10). As with the arbitrary object, the manipulation without sticky finger is slower than the manipulation with sticky fingers. Indeed, some finger repositioning occurs as depicted in Fig.10c
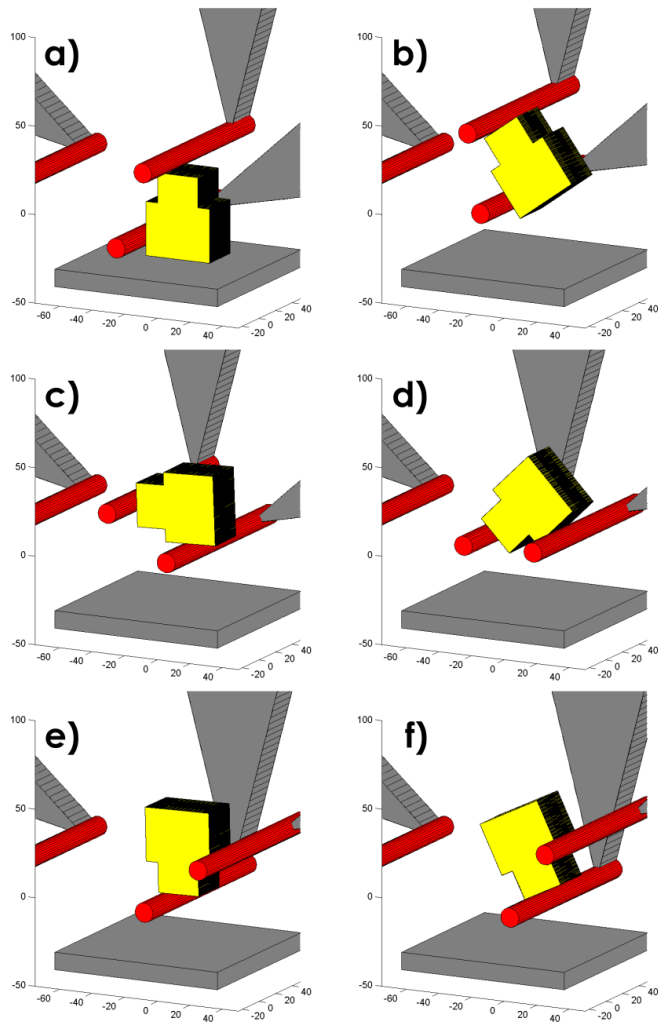

Fig. 8: Images sequence describing the trajectory generated by the planner for a $206^{\circ}$ rotation with sticky fingers on a polygonal object: a) represents the initial grasp. b) to f) show the rotation steps.

and Fig.10e. In the first step, a finger must be placed on the object because the limits of the friction cone are reached with the two first fingers. In the second step, the finger is removed because of the vertex of the shape.

\section{Impact of Adhesion Forces on Manipulation}

1) Impact of the presence of adhesion forces: The differences between the trajectories with and without sticky fingers are due to the stabilizing effect of the adhesion forces. Fig.11 shows this effect on the $M_{2}$ map of the arbitrary shaped object presented in Fig.7 and Fig.9. The blue and yellow areas correspond respectively to the $M_{2}$ maps with and without the stickiness effect. As predicted, the stability area is significantly larger when pull-off forces are considered. This means that there are more options to manipulate the object in presence of adhesion forces.

Moreover, Fig. 12 represents similar results for a re-grasping map in $D_{3}$. It can be seen that, without adhesion, $100 \%$ of the equilibrium nodes in $M_{2}$ (yellow part in Fig.11) are stable re-grasping nodes in $D_{3}$ (yellow part in Fig.12). Conversely, with sticky fingers, the admissible re-grasping nodes (blue part in Fig.12) represent only around $18 \%$ of the equilibrium nodes (blue part in Fig.11). This difference is due to the fact that adhesion which acts as a stabilizing force acts also as a disturbing force when a finger is detached from the object. The (two) remaining fingers must resist to this force which is not possible in all the equilibrium grasps. 

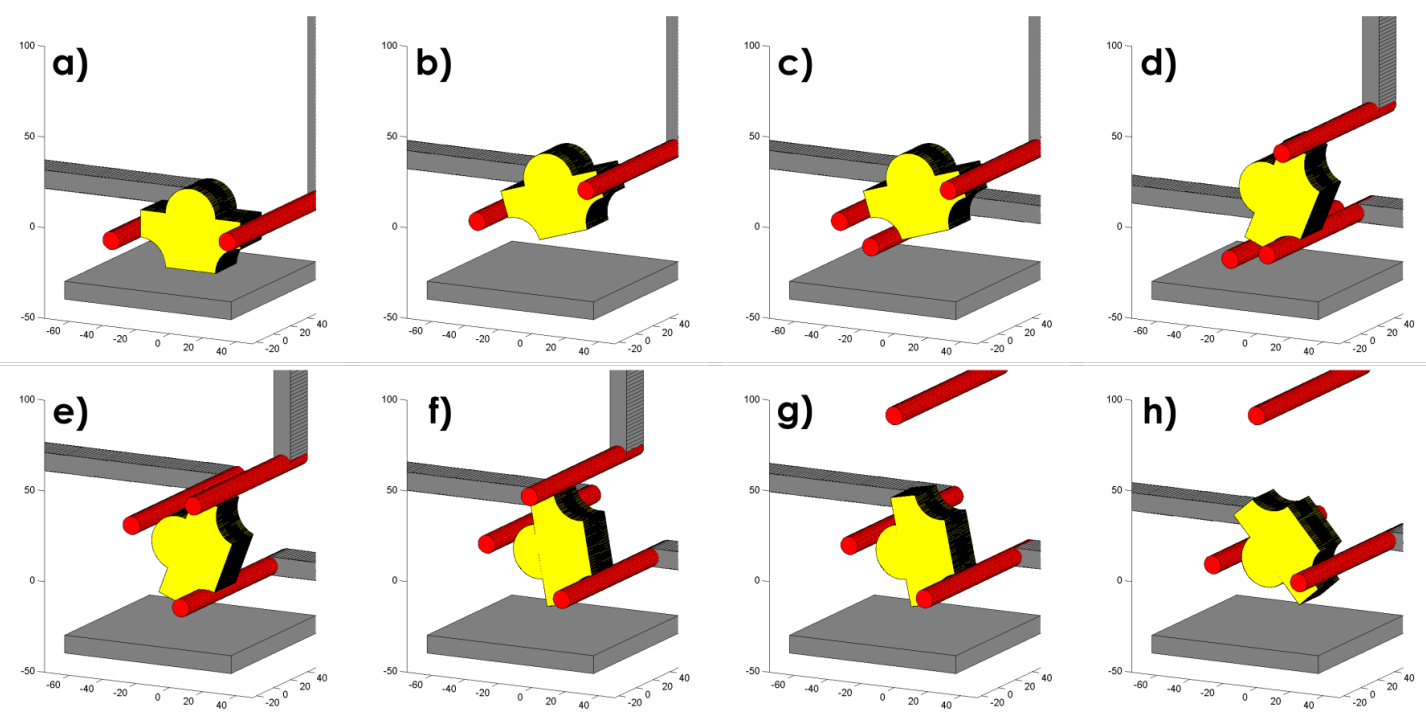

${ }^{100}$ h)

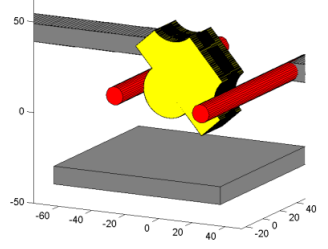

Fig. 9: Images sequence describing the trajectory generated by the planner for a $132^{\circ}$ rotation with non-sticky fingers on an arbitrary shaped object: a) represents the initial grasping configuration while b), d), f) and h) represent the rotation steps. Three finger gaiting steps occur in c), e) and g). In the first step the re-grasping is done to pass through the friction cone's limits while for the others the re-grasping is done to avoid vertices.
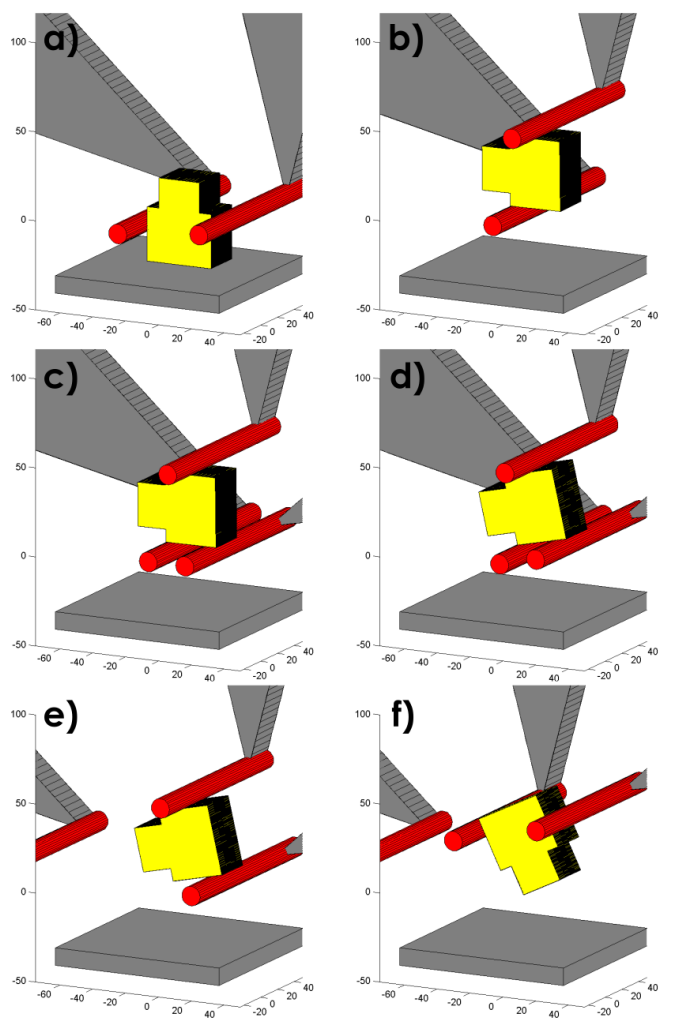

Fig. 10: Images sequence describing the trajectory generated by the planner for a $206^{\circ}$ rotation with non-sticky fingers on a polygonal object: a) represents the initial grasping configuration while b), d), f) represent the rotation steps. Two finger gaiting steps occur in c) and e). In the first case the re-grasping is done to pass through the friction cone's limits while for the second case, the re-grasping is done to avoid the vertex.
2) Impact of the strength of adhesion forces: The presence of adhesion has a strong impact on the trajectories and on the maps but it is important to also evaluate the impact of its strength. Indeed, in the previous examples, various adhesion forces have been used ( $0.6 \mu \mathrm{N}$ in Fig.7 and $1.5 \mu \mathrm{N}$ in Fig.8).

In fact, whether for an adhesion value of $0.5 \mu \mathrm{N}, 5 \mu \mathrm{N}$ or $10 \mu \mathrm{N}$, the maps (equilibrium and re-grasping) are identical as long as the adhesion strength is predominant over the external wrenches. This means that the value of this parameter will have no impact on the stable grasps (maps) and thus on the generated trajectories. For the remaining of this paper, we assume that the external forces are always negligible compared to adhesion forces. More details on this topic can be found in [31].

\section{Sticky Fingers vs Non-Sticky Fingers}

The previous trajectories seem to be more efficient when adhesion forces are exploited. Indeed, on the arbitrary shaped object and on the polygon, the trajectories require less finger gaiting steps and also less displacements of the fingers. A statistical analysis has been done to confirm that manipulation exploiting adhesion forces are better. Trajectories were generated with and without sticky fingers for the two objects presented above and with the same simulation parameters. In every test, the initial grasping configuration is chosen randomly in the admissible initial grasps using two fingers. Moreover, note that with random initial grasp, it is possible that the algorithm does not converge to the desired rotation.

We chose to consider two values to evaluate the performance. The first one is the cost of the computed trajectory based on the cost function described in the previous section. This value estimates the distance traveled by the fingers during the manipulation and is equivalent to the trajectories' 


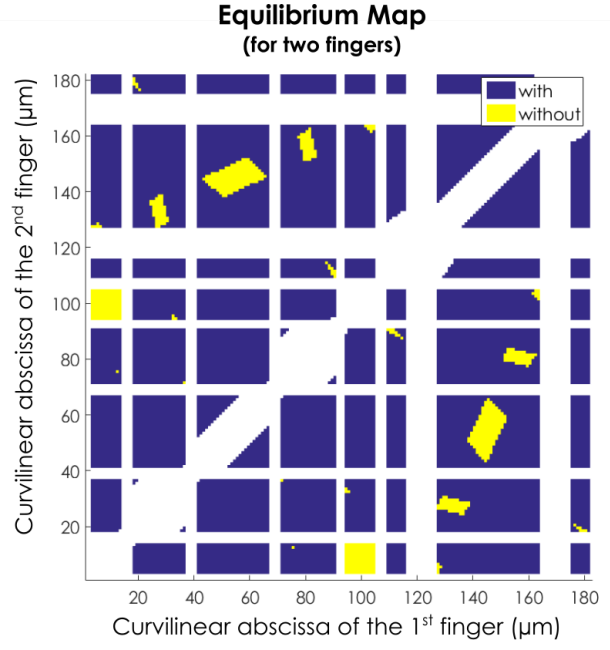

Fig. 11: Representation of the set $M_{2}$ (equilibrium map) with and without pull-off forces on the tested object. Blue and yellow areas correspond respectively to the $M_{2}$ maps with and without sticky fingers.

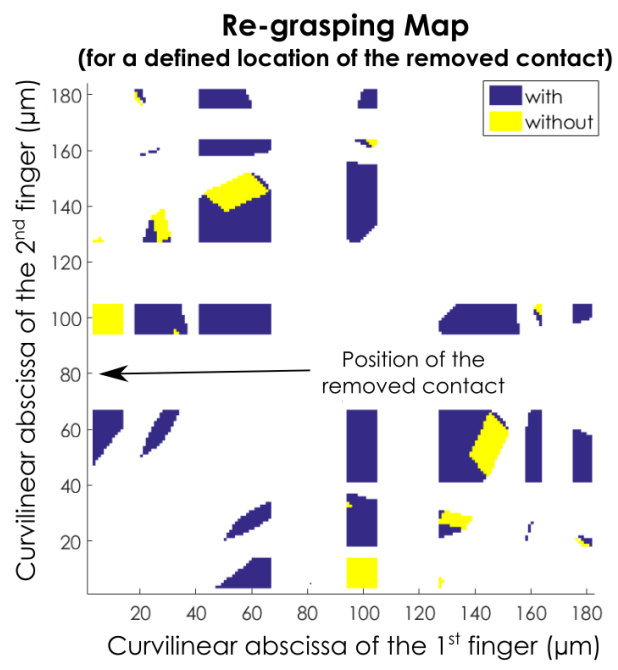

Fig. 12: Representation of the set $D_{3}$ with and without pulloff forces on the arbitrary shaped object. Blue and yellow areas correspond respectively to the $D_{3}$ maps with and without sticky fingers.

execution time. The second one is the convergence rate of the trajectory generation.

1) Arbitrary Shaped Object: Fig.13 shows the statistical results for the arbitrary shaped object. For each rotation amplitude $\left(45^{\circ}, 90^{\circ}, 135^{\circ}, 180^{\circ}\right)$, fifty rotations were generated. It can be seen that, whatever the rotation, the trajectories generated with sticky fingers have an average cost lower than the one generated with non-sticky fingers. This means that less finger gaiting steps are required and that the manipulation process is faster when taking advantage of adhesion forces. Indeed, with sticky fingers, the manipulation of this object is around $40 \%$ faster.

In the case of non-sticky finger, the convergence rate varies from $66 \%$ to $90 \%$ with the highest percentage for the lowest rotation amplitudes. This low convergence rate can be explained by the fact that the hand has only three fingers. Indeed, the re-grasping can occur if and only if two of the three fingers can grasp the object which is very dependent on the object's shape. The convergence rate increase to $95 \%$ in the case of sticky fingers. Therefore, it appears simpler to find an admissible trajectory when adhesion is considered.

2) Polygonal Object: Similar tests have been performed on the polygonal object and the statistical results are depicted in Fig.14. Once again when using sticky fingers, the manipulation is about $35 \%$ faster.

Moreover, the convergence rate varies from $68 \%$ to $84 \%$ for non-sticky fingers whereas the convergence rate is always higher than $90 \%$ with sticky fingers.

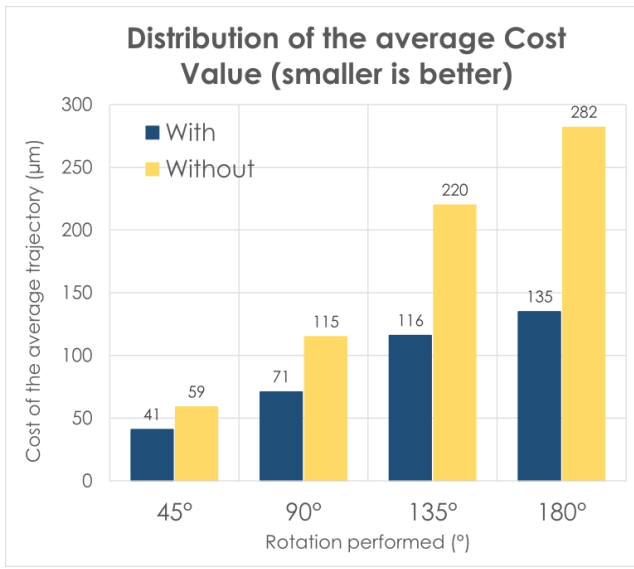

Fig. 13: Chart representing the distribution of the average cost value for the arbitrary shaped object with and without sticky fingers. For each rotation amplitude and each case fifty rotations were generated. The cost value estimates the distance traveled by the fingers during the manipulation.

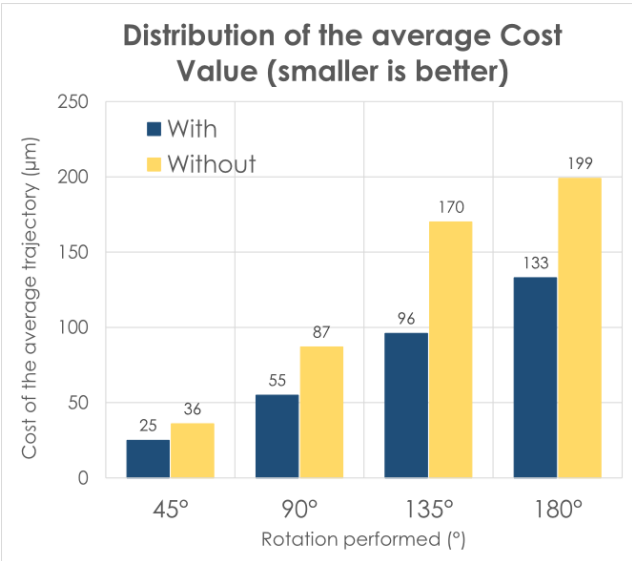

Fig. 14: Chart representing the distribution of the average cost value for the polygonal object with and without sticky fingers. For each rotation amplitude and each case fifty rotations were generated. The cost value estimates the distance traveled by the fingers during the manipulation. 


\section{E. Proof of Concept at Millimeter Scale}

In order to validate this new manipulation strategies, the proposed trajectories exploiting adhesion forces have been tested experimentally. A typical trajectory that does not exploit adhesion forces can be viewed in Fig.1.

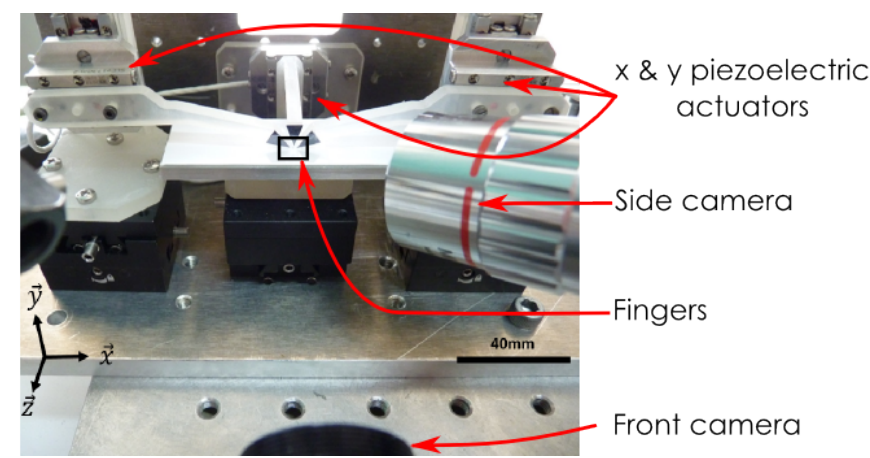

Fig. 15: Photography of the experimental set-up. On this setup, each finger can be attached on a triangular base or on a rectangular base.

1) Experimental Set-up: The experimental set-up (Fig. 15) consists of three cylindrical fingers of $1 \mathrm{~mm}$ diameter made of steel, each one mounted on a 2 Degrees of Freedom translation table. Each table is actuated using 2 Smaract ${ }^{\circledR}$ SLC-1730 piezoelectric actuators with a repeatability of $1.5 \mu \mathrm{m}$. The scene is observed using two high resolution (2560 2018 pixels) cameras from $\mathrm{JAI}^{\circledR}$.

The manipulated objects are made of acrylic and have the following dimensions: $6 \mathrm{~mm}$ long and $4 \mathrm{~mm}$ tall for the object depicted in Fig.16 and $4 \mathrm{~mm}$ long and $4.5 \mathrm{~mm}$ tall for the object depicted in Fig.18.

As this experimental setup is at millimeter scale, so adhesion forces are not predominant. Thus to exhibit the behavior that is encountered in micro-scale, a polymer was deposited on the fingers to enhance the adhesion forces. It generates a behavior which is not identical to the micro-scale behavior but almost similar. Moreover, in the Section V-C, we highlight that the adhesion strength has no impact on the stable grasps and finger gaiting configurations. Thus, even if this polymer does not accurately mimic the micro-world, it is sufficient to fall back to micro-scale specificity where adhesion forces are predominant over other external forces.

The trajectories being generated in the object frame and executed without feedback, the manipulation system must be well calibrated. Thus the homography between the camera frame, the object frame and the actuators frame are computed. Thanks to these homography matrices the computed trajectory for each finger is converted in the actuators frame and executed.

2) Experimental Validation: The trajectories presented previously have been tested on the experimental set-up. Fig.16 represents an images sequence which shows the achievement of the $132^{\circ}$ rotation for the arbitrary shaped object. It can be seen that this manipulation process is successfully executed. The reference trajectory and the real one obtained using the ESM visual tracking algorithm [32] are shown in Fig.17. It can be noticed that the angle of the manipulated object reaches $129.64^{\circ}$ which represents an error of $1.79 \%$. We can see from the same figure the evolution of the position of the manipulated object during the manipulation.

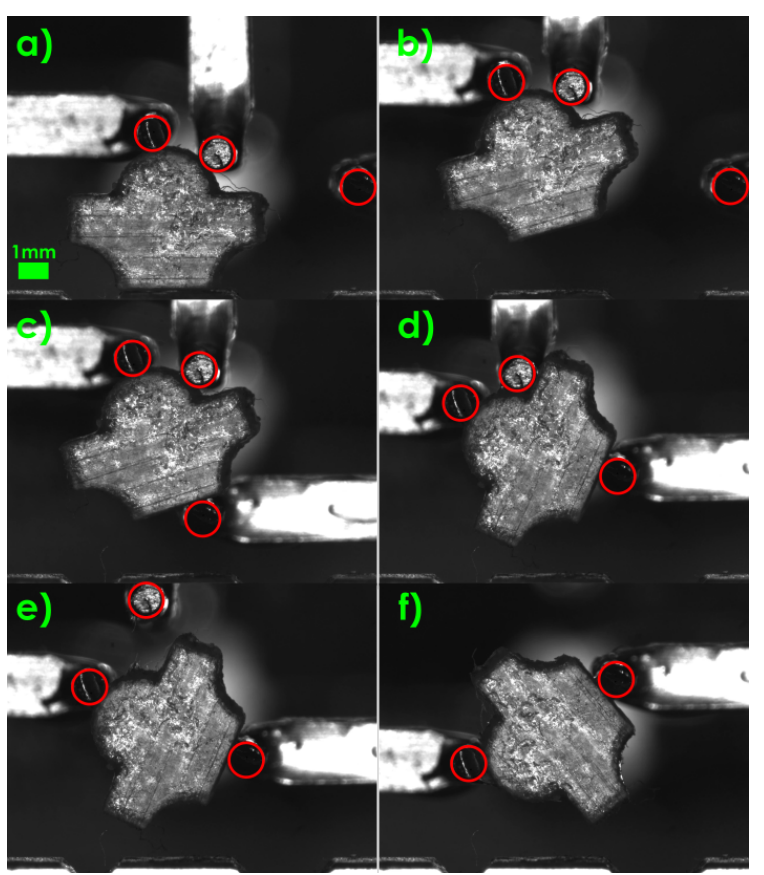

Fig. 16: Image sequence captured during the experimental validation of a $132^{\circ}$ rotation: a) represents the initial grasping configuration while b) to f) represent the rotation steps. A finger gaiting step occurs in e) to avoid a multiple contact configuration between the object and the finger. Fingers are highlighted by the red circles.
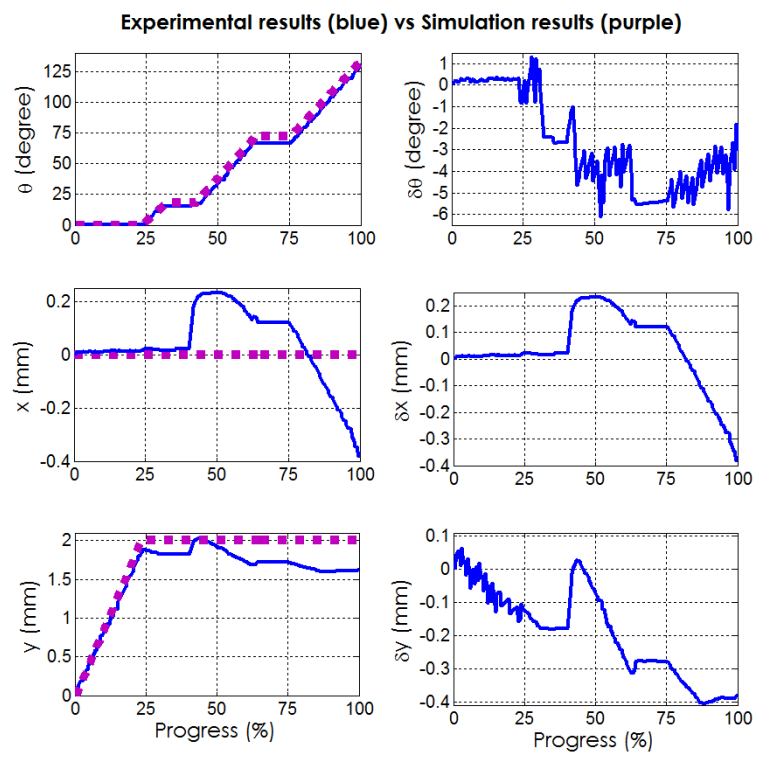

Fig. 17: Representation of the position and orientation of the manipulated object during the experimental validation of the $132^{\circ}$ rotation. Left graphics compare the reference trajectory with the executed one. Right graphics show the various errors during the process. 


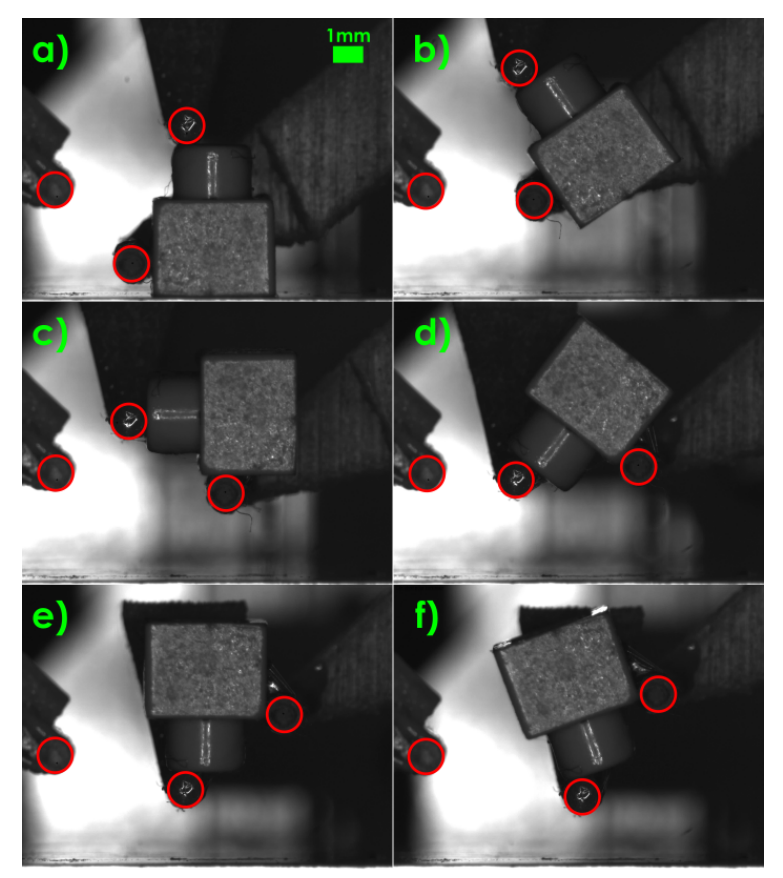

Fig. 18: Image sequence captured during the experimental validation of a $206^{\circ}$ rotation: a) represents the initial grasping configuration while $b$ ) to $f$ ) represent the rotation steps. Fingers are highlighted by the red circles.
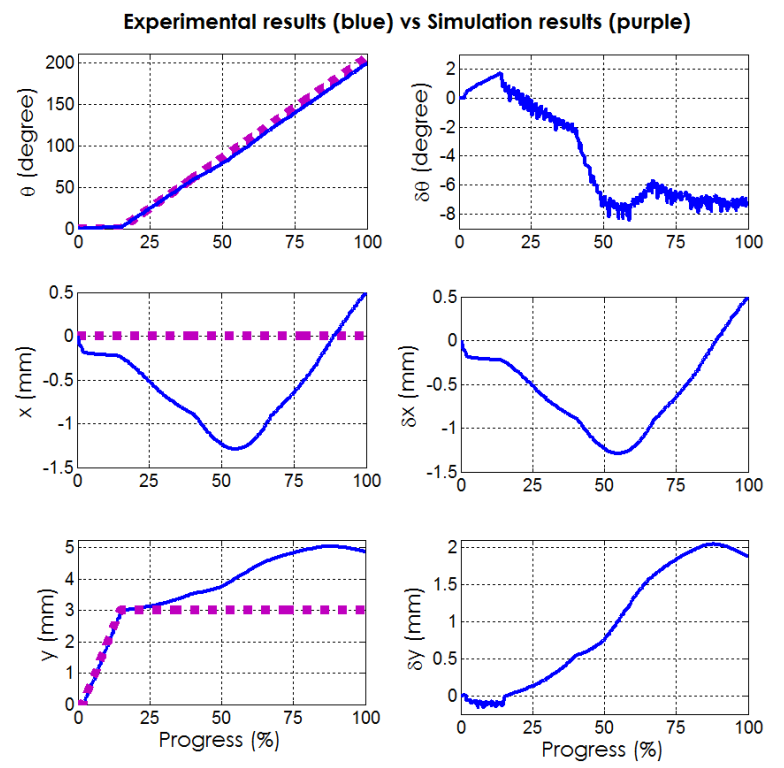

Fig. 19: Representation of the position and orientation of the manipulated object during the experimental validation of the $206^{\circ}$ rotation. Left graphics compare the reference trajectory with the executed one. Right graphics show the various errors during the process.

The experiment has been reproduced with the polygonal object. The experimental results are displayed in Fig.18. Again, it can be seen that the desired motion is successfully achieved thanks to the sticky fingers. The differences between the computed trajectory and the real one are shown in Fig.19.
The rotation angle of the manipulated object reached in this case $199.25^{\circ}$ resulting in an error of $3.6 \%$

In both cases, the errors can be explained by the fact that the finger and the object characteristics (dimension, stiffness, ect.) are not perfectly known. Nevertheless, these errors can be reduced using a classical visual feedback control.

Moreover, it is interesting to note that the trajectories used to moved the object depicted in Fig.18 were generated for a planar object (as depicted in Fig.8). However, the real object have a shape similar to the LEGO's shape (a square with a cylinder on the top). This means that it is possible to manipulate more complex objects as far as the contact points have the same profile.

\section{DISCUSSION}

\section{A. Trajectory optimization}

In the trajectories presented above, the initial grasp was selected randomly among all the possible stable grasps. This means that the optimization performed by the planner is constrained by this initial choice which is unlikely the most optimal. Although, it is theoretically possible to generate the manipulation trajectories for all the possible grasps and select the best one. However, the additional computation time has to be shorter than the time saved in the optimal trajectory execution, which is not the case yet. To do so, the generation time of the initial grasp and manipulation trajectories has to be optimized.

\section{B. Planar manipulation}

The problem of dexterous manipulation using adhesion forces has been formalized. However, the algorithm was developed and the data were generated for planar manipulation. One possible approach to extend the proposed method to 3D manipulation is to perform successive 2D rotations. In this case, the fingers shape has to be modified (sphere, probes, etc...) since cylinders would not be suitable. If the contacts rolling is not negligible as it was the case in this paper then the nonholonomic constraint has to be taken into account.

\section{Adhesion forces and friction coefficient}

The proposed approach relies on three assumptions: the existence of adhesion forces, an estimation of the adhesion strength and an estimation of the friction coefficient.

As shown in [31], as long as the adhesion force is predominant compared to external forces, finger trajectories are identical whatever the adhesion strength is.

Moreover, it is well admitted by the micro-manipulation and micro-assembly community that adhesion forces exist and are predominant in the micro-scale. The trend for the last two decades was to try to get rid of these forces. Since our new paradigm was to take advantage of these forces, if these forces are not strong enough to be exploited, specific chemical treatments can be applied to enhance the adhesion forces [22]. Thus, the uncertainty on adhesion forces can be handle by making sure that they are the predominant forces. In addition, it is also possible to plan the trajectories considering a worst 
case scenario: the rotation are planed considering that each finger is sticky and the re-grasping are planned considering that only the removed finger is sticky. This will force the algorithm to only use a subset of the $D$ maps.

Another precaution can be taken regarding the friction coefficient. Indeed, in this paper various friction coefficient have been used ( 0.3 and 0.26 ). In fact, we chose to take these values to be sure that we never overestimate the friction coefficient. Indeed, never overestimating the friction coefficient ensures that the algorithm will never use a re-grasping node that may lead to instability. We have measured a friction coefficient around 0.7 between the object (made of acrylic) and the fingers (made of steel). Thus the chosen values are acceptable.

\section{Releasing task}

In this paper, we have not studied the releasing task of the micro-objects. In fact, previous works in the literature tried to solve this issue. One interesting way to perform this releasing task is to use the dynamics of the micro-manipulator to overcome the adhesion forces [33] [34]. However, in most industrial micro-assembly tasks, the manipulated object is inserted, welded or glued with another part [35]. In these cases, the adhesion forces between the two parts is higher than the adhesion between the fingers and the object meaning that the releasing can be done easily.

\section{E. Macro-manipulation}

Even if the context of this study was micro-manipulation and micro-assembly, it is possible to imagine extending these results to macro-manipulation. Indeed, the benefit of using sticky finger is independent from the manipulation scale. Thus, considering robotic hands with suction effect at the fingertips may improve the dexterity of the hand. Incidentally, this was a winning strategy during the Amazon Picking Challenge at ICRA 2015.

\section{F. Industrial applications}

The potential applications of this micro-manipulation approach are very large. Indeed, micro-manipulation techniques and more particularly dexterous micro-manipulation can find applications in various fields such as scientific instrumentation and manufacturing [35]. For example several applications in the watch industry require to precisely orientate micro parts to achieve the watch assembly. This is also useful in optics to assemble complex MOEMS (micro-opto-electro-mechanical systems) [36] or optical fibers [25]. Moreover, some other applications can be found in the NOEMS (nano-opto-electromechanical systems) assembly and manipulation [37].

\section{CONCLUSION}

In this paper, a trajectory planner for dexterous micromanipulation was presented. This planner is based on an $A^{*}$ graph search algorithm in order to generate optimal and complete trajectories that take into account the specificity of the micro-scale: the adhesion forces that predominate over gravitational and inertial forces.
The trajectories were validated experimentally and in simulation considering different conditions of use (sticky and non-sticky fingers) and on various object shapes (arbitrary and polygonal shape). These results show that in-hand micromanipulation using adhesion forces can significantly enhance dexterous in-hand manipulation. Thus original trajectories can be generated and executed with a relatively low error.

The next steps of this work will involve the validation of these new trajectories on an experimental micro-manipulation setup (Fig.1). In addition, the current planner will be improved to automatically select the initial grasp to generate globally optimal dexterous in-hand manipulation trajectories and will be extended to non-planar objects. Visual feedback control will also be considered to compensate for the manipulation errors. Furthermore, taking into account the temporal aspect of the finger placement (especially during the initial grasp) will be investigated as well as different model of the contact finger-object.

\section{ACKNOWLEDGMENT}

This work was supported by ACTION, the French ANR Labex no. "ANR-11-LABX-01-01" (http://www.labex-action.fr), by the Equipex ROBOTEX project (contract "ANR-10-EQPX-44-01") and by the Conseil Régional de Franche-Comté.

\section{REFERENCES}

[1] A. M. Okamura, N. Smaby and M. R. Cutkosky, "An overview of dexterous manipulation," in International Conference on Robotics and Automation, pp. 255-262, 2000.

[2] Z. Li, J. Canny and S. Sastry, "On motion planning for dexterous manipulation. i) the problem formulation," in International Conference on Robotics and Automation, pp. 775-780, 1989.

[3] A. Bicchi and R. Sorrentino, "Dexterous manipulation through rolling,", in International Conference on Robotics and Automation, pp. 452-457, 1995.

[4] D. L. Brock, "Enhancing the dexterity of robot hand using controlled slip," in International Conference on Robotics and Automation, pp. 249251, 1988.

[5] L. Han and J. C. Trinkle, "Dexterous manipulation by rolling and finger gaiting," in International Conference on Robotics and Automation, pp. 730-735, 1998.

[6] H. Xie and S. Régnier, "Three-dimensional automated micromanipulation using a nanotip gripper with multi-feedback," Journal of Micromechanics and Microengineering, vol. 19, no. 7, 2009.

[7] H. K. Chu, J. K. Mills and W. L. Cleghorn, "Dual-arm micromanipulation and handling of objects through visual images," in International Conference on Mechatronics and Automation, pp. 813-818, 2012.

[8] J. T. Feddema, P. Xavier and R. Brown, "Micro-assembly planning with van der waals force,"Journal of Micromechatronics, vol. 1, no. 2, pp. 139-153, 2001

[9] H. Saijo, M. Kojima, M. Kamiyama, Y. Mae and T. Arai, "Development of thermos responsive gel coated end effector mircro manipulation," in International Conference on Intelligent Robots and Systems, pp. 189194, 2015.

[10] D. J. Cappelleri, P. Cheng, J. Fink, B. Gavrea and V. Kumar, "Automated assembly for mesoscale parts," in Transactions on Automation Science and Engineering, vol. 8, no. 3, pp. 598-613, 2011.

[11] Q. Zhou, P. Korhonen, J. Laitinen and S. Sjövall, "Automatic dextrous microhandling based on a 6-dof microgripper," Journal of Micromechatronics, vol. 3, no. 3, pp. 359-387, 2006.

[12] K. Inoue, T. Tanikawa and T. Arai, "Micro hand with two rotational fingers and manipulation of small objects by teleoperation," in International Symposium on Micro-NanoMechtronics and Human Science, pp. 97-102, 2008. 
[13] B. Brazey, R. Dahmouche, J. A. Seon and M. Gauthier, "Experimental validation of in-hand planar orientation and translation at microscale," Intelligent Service Robotics, Special Issue on Multi-scale Manipulation Toward Robotic Manufacturing Technologies, vol. 9, pp. 101-112, 2015.

[14] L. U. Odhner and A. M. Dollar, "Dexterous manipulation with under actuated elastic hands," in International Conference on Robotics and Automation, pp. 5254-5260, 2011.

[15] A. Sudsang, J. Ponce and N. Srinivasa, "Grasping and in-hand manipulation: Experiments with a reconfigurable gripper," Advaned Robotics, vol. 12, no. 5, pp. 509-533, 1997.

[16] N. Tan, C. Clévy, G. J. Laurent, P. Sandoz and N. Chaillet, "Characterization and compensation of xy micropositioning robots using vision and pseudo-periodic encoded patterns," in International Conference on Robotics and Automation, pp. 2819-2824, 2014.

[17] R. S. Fearing, "Survey of sticking effects for micro parts handling," in International Conference on Intelligent Robots and Systems, pp. 212 217, 1995.

[18] M. Savia and H. N. Koivo, "Contact micromanipulation survey of strategies," Tronsaction on Mechatronics, vol. 14, no. 4, pp. 504-514, 2009.

[19] F. Arai, T. Fukuda, H. Iwata and K. Itoigawa, "Integrated micro end effector for dexterous micromanipulation," in Seventh International Symposium Micro Machine and Human Science, pp. 149-156, 1996.

[20] J. Corney, P. Lambert and A. Delchambre, "A study of capillary forces as a gripping principle," Assembly automation, vol. 25, no. 4, pp. 275-283, 2005.

[21] J. Dejeu, M. Bechlany, P. Rougeot, L. Philippe and M. Gauthier, "Adhesion control for micro and nano manipulation," ACS nano, vol. 5, no. 6, pp. 4648-4657, 2011.

[22] A. Cot, J. Dejeu, S. Lakard, P. Rougeot and M. Gauthier, "Modeling of electrostatic forces induced by chemical surface functionalisation for microrobotics applications," in International Conference on Intelligent Robots and Systems, pp. 2065-2070, 2013.

[23] J. A. Seon, R. Dahmouche, B. Brazey and M. Gauthier, "Finger Trajectory Generation for Planar Dexterous Micro-Manipulation," in International Conference on Robotics and Automation, 2016.

[24] J. Thompson and R. S. Fearing, "Automating microassembly with orthotweezers and force sensing," in International Conference on Intelligent Robots and Systems, pp. 1327-1337, 2001

[25] J.B. Zhang, K.K. Lu, W.H. Chen, J. Jiang and W.J. Chen, "Monolithically integrated two-axis microgripper for polarization maintaining in optical fiber assembly," in Review of Scientific Instruments,vol. 82, no. 2, pp. 025105, 2015.

[26] J. D. Wason, J. T. Wen, J. Gorman and J. J. Dagalakis, "Automated multiprobe microassembly using vision feedback," in Transactions on Robotics, vol. 28, no. 5, pp. 1090-1103, 2012.

[27] A. Bicchi, "On the closure properties of robotic grasping," The International Journal of Robotics Research, vol. 14, no. 4, pp. 319-334, 1995.

[28] J. Ponce and B. Faverjon, "On computing three-finger force-closure grasp of polygonal objects," Transaction on Robotics and Automation, vol. 11, no. 6, pp. 868-881, 1995.

[29] Y. Wu, D. Sun and W. Huang, "Force and motion analysis for automated cell transportation with optical tweezers," in 9th World Congress on Intelligent Control and Automation, pp. 839-843, 2011.

[30] D. J. Cappelleri, M. Fatovic and U. Shah, "Caging micromanipulation for automated microassembly," in International Conference on Robotics and Automation, pp. 3145-3150, 2011.

[31] J. A. Seon, R. Dahmouche and M. Gauthier, "On the Contribution of Adhesion and Friction in Planning Dexterous in-Hand Micromanipulation," in Journal of Micro-Bio Robotics, Special Issue MARSS 2016, 2017.

[32] S. Benhimane and E. Malis, "Homography-based 2d visual tracking and servoing," in The International Journal of Robotics Research, vol. 26, no. 7, pp. 661-676, 2007.

[33] T. Chen, M Pan, Y. Wang, J. Liu, L. Chen and L. Sun, "Manipulation of microobjects based on dynamic adhesion control," in International Journal of Advanced Robotic Systems, vol. 9, 2012.

[34] DS. Haliyo and S Régnier, "Advanced applications using [mu] MAD, the adhesion based dynamic micro-manipulator," in International Conference on Advanced Intelligent Mechatronics, vol. 2, pp. 880-885 2003.

[35] M. Gauthier, C. Clévy, D. Hériban and P. Kalio, "Industrial Tools for Micromanipulation," in Micro-and Nanomanipulation Tools, vol. 13, 2015.

[36] K. Rabenorosoa, C. Clévy, P. Lutz, A. Das, R. Rakesh and D. Popa, "Precise motion control of a piezoelectric microgripper for microspectrometer assembly," in International Design Engineering Technical Con- ferences and Computers and Information in Engineering Conference, pp. 769-776, 2009.

[37] C. Shi, D.K. Luu, Q. Yang, J. Liu, J. Chen, C. Ru, S. Xie, J. Luo, J. Ge and Y. Sun, "Recent advances in nanorobotic manipulation inside scanning electron microscopes," in Microsystems \& Nanoengineering, vol. 2, 2016.

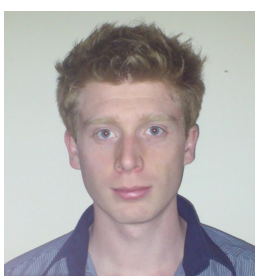

Jean-Antoine Seon is a Postdoctoral fellow at the Aalto University (Espoo, Finland) in the micro-nano robotics group. He obtained a Ph.D. in Automation and Robotics from the University of Bourgogne Franche-Comté (Besançon, France) in 2017 and a Master degree in mechatronics and micro-systems from ENSMM (Besançon, France) in 2014. His research interests have been focused on optimal planning and control for both contact and contactless micro-manipulators.

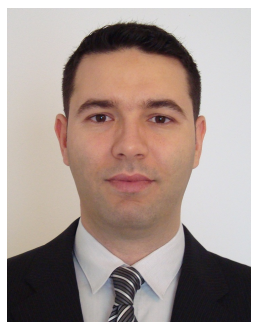

Redwan Dahmouche is an Associate Professor at the University of Bourgogne Franche-Comté', and a researcher at the femto-st Institute in France. He obtained an Electrical Engineering, a Master's, and a $\mathrm{Ph} . \mathrm{D}$ degrees respectively in 2005, 2006, and 2010. During the last decade, he has been working on different fields of robotics such as vision based control, parallel robots, and cable driven robots. Currently, his main research topics are medical robotics, micro/nano-robotics, and dexterous manipulation. Beside his academic research activities, he is also involved in know-how transfer into the industry, where he is the head of several technology transfer projects, and the co-author of several patents.

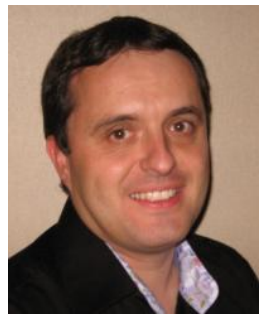

Michaël Gauthier is a CNRS senior scientist (eq full professor) and the vice-head of FEMTO-ST institute, Besanon, France. He works in the field of micro-nanorobotics since 2000 and has proposed, modeled and experimented new robotics tools for microhandling and micro-assembly in several European and National projects. He is the author of 2 books, more than 35 papers and 55 conference talks. He is the co-founder of the start-up Percipio Robotics providing micro-assembly platforms for industrial applications since 2011. He is currently focusing their works on micro-nano-robots based on non-contact forces and dexterous microgrippers. . 